\title{
Remarques sur l'espèce Asellus cavaticus Leydig (Crustacé Isopode hypogé) et description d'une sous-espèce nouvelle \\ Par Jean-Paul Henri ${ }^{1}$ )
}

Asec planches 9 (1) et 10 (2)

Avant les travaux de Racovitza en 1919 sur le genre Asellus, on ne distinguait en Europe que deux espèces : Asellus aquaticus L. qualifiant les formes épigées, Asellus cavaticus Leydig dénommant les Aselles hypogés ${ }^{2}$ ). Depuis, les descriptions d'espèces nouvelles sont devenues très nombreuses et démontrent que le genre Asellus est spécifiquement beaucoup plus différencié qu'on ne le pensait.

Le nombre toujours croissant de nouvelles formes décrites incita les auteurs à rechercher les différentes lignées évolutives et à réunir les espèces en sous-genres ou en "groupes». C'est ainsi que pour les Aselles cavernicoles du sous-genre Proasellus, défini par Dudich en 1925, Chappuis (1953) reconnaît trois lignées ayant chacune une aire de répartition propre : la lignée de coxalis dont la distribution géographique est circum-méditerranéenne, celle d'anophthalmus répandue dans les Balkans et enfin celle de cavaticus qui s'étend de l'Angleterre jusqu'en Autriche.

Les deux premières lignées, dont les premiers représentants troglobies n'ont été découverts respectivement qu'en 1922 (A.spelaeus Racovitza) et 1934(A. anophthalmus Karaman), n'en sont pas moins représentées actuellement par plusieurs espèces et sous-espèces. Il en va bien différemment de la lignée de cavaticus qui n'était connue jusqu'en 1963 que par la seule espèce qui lui donne son nom, Asellus casaticus, pourtant découverte depuis 1871 et baptisée par Schiödte puis décrite som-

1) Laboratoire de Biologie animale et générale, Faculté des Sciences, Dijon.

$\left.{ }^{2}\right)$ En 1876, de Rougemont avait bien décrit un nouvel Aselle aveugle des eaux souterraines, $A$. Sieboldi, qui fut reconnu comme synonyme de cavaticus; puis en 1879, Blanc avait publié une description d'une nouvelle espèce, A. Foreli, des profondeurs du Lac Léman, mais qui n'était qu'une forme très voisine de cavaticus. 
mairement par Leydig en 1878 d'une grotte du Wurtemberg. On pensait devant les nombreuses récoltes d'Aselles cavernicoles faites en Europe que cette lignée était homogène et qu'elle ne renfermait qu'une seule forme.

Avant Racovitza cette manière de voir s'expliquait assez bien. Ne reconnaissant qu'une seule forme d'Aselle cavernicole, les auteurs rapportaient à cette espèce tous les Aselles du domaine souterrain. Malgré une description assez détaillée d'Asellus cavaticus que publia Miethe en 1899, les déterminations restaient basées sur des diagnoses aussi imparfaites et incomplètes que celles s'appuyant sur la coloration blanche et l'absence d'yeux. Un exemple frappant de ces erreurs traditionnelles est fourni par Viré (1904) lors de son étude de la faune du Gouffre de Padirac. Ayant recueilli à la fois des Aselles pigmentés et oculés et d'autres «décolorés et complètement aveugles» il rapporte les premiers à Asellus aquaticus et constate que les seconds «répondent parfaitement au type de l'Asellus cavaticus de Schiödte». Mais la présence dans ses récoltes d'individus présentant tous les intermédiaires entre ces deux formes fait même croire à l'auteur qu'il n'existe qu'une seule espèce d'Aselle, A. aquaticus, «modifiable selon les milieux où il vit». On sait que par la suite Racovitza a reconnu qu'il s'agissait en réalité d'une espèce nouvelle, Asellus meridianus, bien distincte de l'A. aquaticus.

Après les travaux de ce dernier auteur qui montrèrent l'intérêt d'utiliser l'appareil copulateur mâle comme caractère spécifique, les chercheurs examinèrent plus attentivement les Aselles connus sous le nom d'A.cavaticus et provenant de différentes stations. On publia alors de nouvelles et plus complètes descriptions de cette espèce. Tattersall (1930) décrivit en détail des exemplaires d'Angleterre et Stammer (1932) examina des individus de la station-type et d'autres de Strasbourg.

Ces études pourtant plus minutieuses ne permirent pas aux chercheurs de reconnaître dans cette lignée différentes formes et elle continua à n'être représentée que par une seule et unique espèce que l'on considéra comme très ubiquiste. Cette opinion s'affirma malgré les tentatives malheureuses qui furent néanmoins faites pour créer des formes infraspécifiques de l'espèce cavaticus. Karaman décrivit successivement en 1933 et 1934 les sous-espèces hercegovinensis et montenigrinus des Balkans, puis Arcangeli (1935) créa la sous-espèce leruthi pour les cavaticus des cavernes de Belgique. Or les formes slaves de Karaman appartiennent à la lignée d'anophthalmus et n'ont pas d'af- 
finité avec celle de casaticus ${ }^{3}$ ), quant à leruthi il ne diffère des autres spécimens de cavaticus que par de très petites différences, comme celles que l'on peut rencontrer entre des individus de diverses stations.

Alors que les lignées de coxalis et d'anophthalmus s'enrichissent de plusieurs espèces et sous-espèces bien définies, il faut attendre 1948 pour rencontrer des populations d'Aselles, se rangeant dans la lignée d'Asellus cavaticus, mais dont les caractères morphologiques s'écartent trop de ceux des exemplaires typiques de cette espèce pour que l'on puisse en faire de simples Asellus cavaticus. Chappuis étudiant ces captures a jugé les divergences assez importantes pour considérer ces populations comme des sous-espèces distinctes et il décrivit ainsi trois sous-espèces des eaux souterraines suisses : A. casaticus foreli, A.cavaticus walteri et A.casaticus valdensis.

Cet auteur qui continua à étudier cette espèce prit peu de temps après ses trois descriptions une attitude surprenante : il renonca à nommer les différentes formes d'A.casaticus qu'il aurait éventuellement à examiner, pensant que dans une aire de distribution aussi vaste que celle de cavaticus les différentes formes ne se distingueraient les unes des autres que par des petites différences seulement et qu'il devenait superflu de leur donner un nom spécial.

Cette prise de position telle qu'elle est exposée par l'auteur est conforme aux idées émises par ceux qui l'ont précédé dans l'étude systématique d'A.cavaticus. Personne bien entendu ne doit créer à tous prix de nouvelles sous-espèces basées sur des caractères très minimes qui ne sont peut-être dus qu'à des variations individuelles. Mais une fois que des critères distinctifs fondamentaux ont été reconnus, il est peu concevable de renoncer à nommer de nouvelles formes ce qui, à notre avis, arrêterait toute tentative d'explication de l'histoire phylétique de la lignée de casaticus. Il faut souligner que Chappuis suivant en cela Racovitza (1919) et Braga (1948) prend bien soin justement de définir ces critères taxonomiques fondamentaux : forme et structure de l'endopodite du pléopode II du mâle ; il s'ensuit qu'il est alors forcé, en présence de certaines populations d'A.casaticus, de reconnaître des sous-espèces mais ne voulant pas les nommer, il les indique seulement comme : A.cavaticus subsp. de la grotte des Foules, A.casaticus subsp. de Küssaberg, etc. (Chappuis 1953). Nous ne croyons pas qu'une telle méthode soit possible; elle ne cache que notre incompétence à reconnaître l'évolution de la lignée en cause et ne sert pas la systématique.

Karaman eut beaucoup moins de scrupule en nommant en 1955 une sous-espèce autrichienne strouhali d'après les schémas des extrémités

3) L'auteur les a d'ailleurs récemment (1955) élévées au rang d'espèces. 
caractéristiques que Chappuis avait publiés dans son travail de 1949. Cette espèce fut par la suite étudiée en détail sur du matériel provenant de Salzbourg par Strouhal (1957) qui en conclusion de sa description donne son avis sur l'orientation des futures recherches systématiques sur l'espèce cavaticus : «... daß künftige systematische Untersuchungen von cavaticus sich in erster Linie auf die 1., 2. und 3. Pleopoden zu erstrecken haben, wobei in ganz besonderem Maße die Endorgane der 2. Pleopoden-Endopoditen des Männchens eine bis ins Detail gehende Behandlung erfahren müssen.»

Dès 1951, Remy qui estimait, lui aussi, que les pléopodes II du mâle fournissaient un bon caractère, avait rapporté à la sous-espèce valdensis les A.cavaticus recueillis par Sollaud (1932 et 1933) à la résurgence du Bief Noir (Jura), et pensait qu'il fallait y rattacher également ceux de la grotte de la Balme découverts par Léger (in Jeannel 1926) car les organes copulateurs des mâles sont semblables.

Ayant eu à déterminer des Aselles souterrains, nous nous sommes donc particulièrement attachés à considérer les critères proposés par les auteurs cités ci-dessus, tous unanimes à reconnaître la valeur systématique du dispositif terminal de l'endopodite des pléopodes II des mâles. C'est ainsi que nous avons été amenés à créer la nouvelle espèce Asellus synaselloides Henry 1963 appartenant à la lignée de cavaticus pour des individus d'une grotte de Basse-Provence et que nous décrivons ci-dessous une nouvelle sous-espèce de casaticus pour des spécimens d'un puits du Beaujolais. Une autre récolte ${ }^{4}$ ) faite au Plateau de l'Ile Crémieu (Isère) est constituée d'individus que nous déterminons comme se rapportant à la sous-espèce valdensis; cependant ayant relevé quelques petites différences entre la description assez incomplète du spécimen-type de la sous-espèce et nos individus, nous donnons ici une description plus détaillée et plus illustrée de nos exemplaires. Nous avons pris comme type de référence pour casaticus des exemplaires de la grotte de Sainte-Reine près de Toul (Meurthe-etMoselle). Deux raisons ont guidé notre choix. D'une part, les Aselles de Sainte-Reine sont connus depuis 1904 et ont toujours été considérés comme très voisins, sinon identiques au type. D'autre part, cette espèce est très répandue dans l'Est de la France, où elle a été trouvée en de nombreuses cavités, tant naturelles qu'artificielles (Husson 1938, Remy 1948).

4) Ces deux dernières récoltes ont été effectuées en 1964 par Monsieur Daniel Ariagno de la Faculté des Sciences de Lyon qui nous les a obligeamment communiquées. 
ASELLUS CAVATICUS PUTEANUS n.subsp. $\left.{ }^{5}\right)$

(Planche 1)

Station : Les animaux (10 mâles et 10 femelles dont une à poche incubatrice vide) ont été capturés dans un puits situé sur la commune du Pérréon, au hameau de la Creuze, à $15 \mathrm{~km}$. au Nord-Ouest de Villefranche-sur-Saône (département du Rhône).

Dimensions : Le plus grand mâle : longueur $=4,8 \mathrm{~mm}$, largeur $=$ $0,9 \mathrm{~mm}$., antennes $=3,5 \mathrm{~mm}$., pléotelson $=1,3 \mathrm{~mm}$.

La plus grande femelle : longueur $=5,7 \mathrm{~mm}$., largeur $=1,2 \mathrm{~mm}$, antennes $=3,8 \mathrm{~mm}$, pléotelson $=1,4 \mathrm{~mm}$.

Coloration : Blanche; yeux absents.

Antennules: Comportant 11 articles chez les mâles, 9 à 10 chez les femelles; fouet plus long que la hampe, avec 4 lames olfactives chez les mâles, 3 chez les femelles (fig. A), insérées sur le bord distal des articles terminaux, parfois sur le dernier. Chez les individus d' $A$. cavaticus de la grotte de Sainte-Reine, ces organes sensoriels sont au nombre de 6 chez les mâles et de 5 chez les femelles.

Antennes : Plus longues que la moitié du corps chez les deux sexes, elles ont un fouet de 45 articles chez les mâles examinés, et de 35 à 45 chez les femelles.

Pièces buccales : Sans différence notable avec celles des spécimens lorrains. A la mandibule gauche, le lobe porte 10 soies semi-pennées, l'apophyse dentaire 5 dents apicales comme chez la forme-type dont la lacinia mobilis montre 3 dents au lieu de 4 chez puteanus. A la mandibule droite, le lobe porte 11-13 soies semi-pennées au lieu de 15 chez les cavaticus de Sainte-Reine, dont l'apophyse dentaire est identique à celle de notre sous-espèce. La lame interne de la maxille I montre au bord distal les 5 tiges pennées caractéristiques du sousgenre Proasellus. Chez la femelle à marsupium, présence sur le coxa du maxillipède d'un oostégite avec 9-10 soies plumeuses comme chez la femelle ovigère de cavaticus f. typ.

PÉréiopodes : Le nombre des épines sternales des dactyles est plus faible que chez cavaticus.

Péréio pode I d u ô: Le propode ne porte que 3 phanères ensiformes insérés sur le tiers proximal du bord sternal, au lieu de 4, plus robustes, chez les mâles de Sainte-Reine ; le reste du bord sternal est muni des écailles pectinées communes aux deux formes. Rangée submarginale rostrale de 10 tiges courtes et rangée submarginale caudale de 10

5) Du latin: de puits. 
soies. Le dactyle porte une rangée sternale de 4 à 5 épines au lieu de 8 au minimum chez cavaticus ; $6-7$ soies dont 2 ciliées insérées sur le tiers distal du bord dorsal (fig. B).

Péréiopode I de la $q$ : 1 seul phanère ensiforme grêle inséré au milieu du bord sternal du propode, accompagné quelquefois d'une soie plus proximale, alors qu'il y a 3 phanères ensiformes, plus robustes, chez les femelles de Sainte-Reine. La dactyle présente au péréiopode droit 2 épines sternales et 3 au gauche (fig. C) ; chez la femelle de cavaticus, 5 à 6 épines dactyliennes ; 6-7 soies dont 1 ciliée insérées sur le tiers distal du bord dorsal.

Péréiopode IV des deux sexes (fig. D) : Propode rectiligne et assez court, avec une rangée marginale sternale de 3 épines coniques et 2 soies ; bord dorsal avec une rangée marginale de 5 tiges dont 4 ciliées, la plus grande insérée à l'extrémité distale. Dactyle avec 1 seule épine sternale alors qu'il y en a toujours plusieurs chez cavaticus f. typ.

Pléopodes :

Pléopode I du $\widehat{o}$ (fig. E) : Très différent dans ses proportions de celui des cavaticus pris comme type. Sympodite glabre, trapézoïdal, 1 fois $2 / 5$ plus long que large; quadrangulaire, aussi long que large chez la forme type. Bord interne rectiligne plus long que le bord externe légèrement convexe, rétinacle formé de 3 crochets de chaque côté. Exopodite ellipsoïdal, 2 fois $1 / 3$ plus long que large ; subrectangulaire, 1 fois ${ }^{4} / 5$ plus long que large chez cavaticus. L'extrémité distale de cet appendice, très fortement convexe, ne permet plus de distinguer un bord distal alors qu'il est parfaitement reconnaissable chez cavaticus où il est subrectiligne, très légèrement convexe cependant. Une autre différence apparaît dans la répartition des soies sur cet article : chez puteanus 15 soies inégales, non plumeuses, sont insérées sur le tiers terminal du bord externe, la plus distale située exactement à l'apex de l'article marquant ainsi la limite avec le bord interne qui est totalement dépourvu de phanères; en outre, sur la face sternale, 2 petites soies lisses submarginales près de l'extrémité distale et une tige moyenne près de la base de l'exopodite. Chez cavaticus, on observe une rangée de 10-11 soies insérées sur la moitié terminale du bord externe, se continuant sur le bord distal par 6-8 soies lisses ou plumeuses, dont 3-4 toujours sur sa moitié interne.

Pléop ode II du ô (fig. F) : Par son aspect général, cet appendice est analogue à celui des autres cavaticus; mais un examen détaillé de l'endopodite montre que l'organe copulateur de notre forme présente quelques petites différences avec celui de la forme type. Sympodite subquadrangulaire, légèrement plus étroit dans sa partie distale, avec 
3 soies plumeuses submarginales insérées sur la moitié terminale du bord interne. Exopodite légèrement plus long que la moitié du sympodite : article proximal cupuliforme, à bords convexes, sans aucune soie sur son bord externe, à la différence des autres cavaticus où ce bord porte toujours de 1 à 3 soies plumeuses; article distal subtriangulaire à sommet arrondi, un peu plus long que large, 2 fois plus long que l'article proximal; son bord externe porte 5 courtes soies pennées ; au sommet, 2 soies pennées plus longues et une brosse de cils très fins existant aussi chez cavaticus; chez cette dernière, l'extrémité distale porte 3-4 soies plumeuses insérées soit de part et d'autre de l'apex, soit sur la moitié terminale du bord interne. Endopodite comme chez les mâles de Sainte-Reine dans sa forme et ses proportions, seule l'extrémité distale est différente (fig. G). L'apophyse terminale, courte et arrondie, est très nettement recourbée vers l'exopodite et forme avec le goulot ${ }^{6}$ ) un angle de $45^{\circ}$. Ce goulot forme une gouttière étroite, droite sur toute sa longueur, dont les lèvres sont largement béantes sur la plus grande partie de sa longueur. Chez les cavaticus lorrains, l'apophyse, légèrement dirigée vers l'extérieur, forme avec le goulot, très incliné vers l'exopodite, un angle d'environ $90^{\circ}$; le goulot y forme une large gouttière rectiligne.

Plé o p o de I I de la q (fig. H) : De forme triangulaire, oblong, à bord interne rectiligne et à bord externe régulièrement convexe, orné de 7 soies plumeuses.

Pléopode III des deux sexes (fig. I) : Exopodite presque 2 fois aussi long que large, séparé en deux articles par une articulation légèrement oblique. Bord externe de l'article proximal très bombé portant 7 petites soies lisses. Article distal, à bords externe et interne glabres, avec 6 petites soies plumeuses sur la bord distal et 3 courtes soies lisses submarginales vers le bord interne.

Pléo podes IV et V des deux sexes : Sans différence notable avec ceux d'A. cavaticus.

Uropodes : Un peu plus long que la moitié du pléotelson. Les longueurs relatives du sympodite, de l'exopodite et de l'endopodite sont : 1-1,2-1,5, c'est-à-dire sensiblement comme chez cavaticus.

6) Ce terme de goulot a été introduit par Racovitza (1920) qui a comparé la forme de l'organe copulateur du genre Asellus à celle d'une bouteille. Il distingue ainsi le cul-de-bouteille qui correspond à la région proximale de l'endopodite, le corps de la bouteille à la région médiane et enfin le goulot de la bouteille au sommet, plus ou moins recourbé du côté sternal. L'auteur signale que ce goulot «forme le plus souvent une pointe irrégulière... pourvue de deux lèvres onduleuses qui limitent une fente sternale plus ou moins béante». 
Pléotelsox (fig. J) : 1 fois 1/3 plus long que large, il diffère de celui de la forme type par sa forme subrectangulaire et ses bords latéraux subrectilignes. Pointe du telson marquée et arrondie.

Position systématique : Notre sous-espèce appartient au sousgenre Proasellus; la structure de l'extrémité de l'endopodite du pléopode II du mâle nous permet de la classer dans la lignée de cavaticus caractérisée par une apophyse distale et un goulot ayant même direction. C'est par la forme de ce goulot que l'on peut séparer immédiatement puteanus de cavaticus. Le nombre d'épines sternales au dactyle du péréiopode I, la constitution du pléopode I ô et du pléopode III des deux sexes, la forme du pléotelson sont aussi des caractères qui permettent de les distinguer. Cependant nous ne pensons pas devoir attribuer à l'Aselle du Beaujolais le statut d'espèce car par l'aspect du pléopode II $\hat{\sigma}$, c'est de cavaticus qu'elle se rapproche le plus. D'autre part, les caractères des pièces buccales et des pléopodes IV et V sans différence notable chez les deux formes permettent de penser que des liens de parenté très étroits existent entre ces deux troglobies.

\section{ASELLUS CAVATICUS VALDENSIS Chappuis 1948}

(Planche 2)

Station : Les animaux (1 mâle et 4 femelles dont 2 ovigères, l'une à poche incubatrice vide, l'autre avec 33 jeunes) ont été capturés dans la rivière souterraine de la grotte de la Fontaine Saint-Joseph à Verna, canton de Crémieu (département de l'Isère).

Ginet (1953) signale la présence d'A.cavaticus dans cette station. Il est probable qu'il s'agisse en réalité de sa sous-espèce valdensis qui abonde également dans une grotte voisine, celle de la Balme.

Dimensions : Les individus récoltés sont remarquables par leur grande taille. Mâle : longueur $=7,6 \mathrm{~mm}$., largeur $=2,1 \mathrm{~mm}$. au péréionite VII, antennes $=7,7 \mathrm{~mm}$., pléotelson $=1,9 \mathrm{~mm}$.

La plus grande femelle (ovigère) : longueur $=8,5 \mathrm{~mm}$., largeur $=$ $2,4 \mathrm{~mm}$. au péréionite III, antennes $=7,1 \mathrm{~mm}$, pléotelson $=1,9 \mathrm{~mm}$.

Coloration : Blanche; yeux absents.

Antennules : Comportant 10-11 articles chez le mâle et les femelles; fouet plus long que la hampe, avec 3-5 lames olfactives insérées sur le bord distal des articles terminaux sauf le dernier; le nombre de ces organes sensoriels peut ne pas être identique sur les deux antennules.

Antennes: Aussi longues que le corps chez le mâle étudié, plus courtes, mais dépassant la moitié du corps chez les femelles; elles ont un fouet de 51 articles dans les deux sexes. 
PiÈces buccales : Quelques différences apparaissent aux mandibules seules décrites chez le type; à droite, lobe avec 5 dents et 13 tiges au lieu d'une seule dent et 9 tiges chez la forme suisse ; à gauche, le lobe porte 5 dents et 12 tiges au lieu de 2 dents et 9 tiges. Maxilles et maxillipèdes (fig. A) sans différence notable avec ceux des cavaticus pris comme type. Une des femelles ovigères présente aux maxilles I 6 dents à la lame interne.

Péréiopodes : Le dactyle des péréiopodes II à VII est armé de 2 épines sternales alors que chez cavaticus, aux mêmes appendices, il y en a souvent plus de 2 .

Péréio p ode I du ơ: Le propode ne porte que 3 phanères ensiformes au lieu des 4 plus robustes chez les mâles de Sainte-Reine. Le dactyle (fig. B) porte une rangée de 6 épines sternales, la plus distale étant la plus forte, non disposées en une série marginale continue comme le sont les 8 épines dactyliennes chez les mâles de casaticus. Sur le tiers distal du bord dorsal, 6 soies dont 1 ciliée.

Péréiop ode I de la o : Au propode, 3-4 phanères ensiformes grêles insérés sur le bord sternal ; le dactyle (fig. C) porte 4 épines au bord sternal et 8 soies dont 1 ciliée au bord dorsal.

Péréiopode IV des deux sexes (fig. D) : Propode droit, 6 fois aussi long que large, avec une rangée marginale sternale de 5 épines, entre les 4 plus distales, 3 petites soies. Dactyle avec 2 épines sternales.

Pléopodes :

Plé opode I du ô (fig. E) : Il diffère de celui de casaticus f. typ. par la forme de son sympodite et le nombre de crochets de son rétinacle. Sympodite glabre, trapézoïdal, 1 fois $3 / 5$ plus long que large ; sur le bord interne, 1 fois $1 / 3$ plus long que le bord externe, le rétinacle est formé d'un crochet à droite, de 2 à gauche comme chez le type. Exopodite ellipsoïdal, à peine 2 fois plus long que large ; bord distal avec 11 petites soies lisses inégales; bord externe avec 13 soies lisses plus longues insérées dans sa moitié distale; sur la face sternale près de la base, 2 soies moyennes.

Plé op ode II du ô (fig. F) : La forme allongée du sympodite et de l'endopodite donne à cet appendice un aspect très différent de celui que l'on observe chez les mâles de cavaticus. Sympodite subrectangulaire, plus étroit dans sa partie distale, 1 fois $1 / 5$ plus long que large, avec 2 soies plumeuses submarginales insérées sur la moitié distale du bord interne. Exopodite légèrement plus long que la moitié du sympodite : article proximal cupuliforme à bords assez débordants, avec 2 soies pennées au bord interne. Article distal en forme d'ellipse tronquée dans sa région proximale, 1 fois $1 / 2$ plus long que large et presque 3 fois plus long que l'article proximal alors qu'ils sont égaux chez la 
forme suisse. Au bord distal 3 soies pennées et une brosse de cils très fins. Endopodite très différent dans ses proportions de celui des mâles de cavaticus; il est bananiforme, 4 fois plus long que large ; les apophyses proximales des angles internes et externes sont peu développées ; à l'extrémité, si l'apophyse distale est sensiblement de même forme et de même orientation que chez les mâles de Sainte-Reine, la structure du goulot s'en écarte assez profondément. Il est formé chez valdensis (fig. G) par une large gouttière qui se relève fortement vers l'exopodite lui donnant ainsi l'aspect d'un crochet. L'angle formé par le goulot et l'apophyse est ici d'environ $90^{\circ}$.

Plé o p ode I I de la o (fig. H) : Subtrapézoïdal, le bord interne, rectiligne, et les $2 / 3$ proximaux du bord externe sont subparallèles; le tiers distal du bord externe est garni de 6 soies plumeuses insérées à équidistance l'une de l'autre.

Plé op ode I I I des deux sexes (fig. I) : Exopodite de forme ovale séparé en 2 articles par une articulation oblique. Bord distal et bord externe garnis de courtes soies lisses, bord interne glabre. Endopodite très court, ne dépassant pas l'article proximal de l'exopodite.

Plé o podes IV et V des deux sexes: Sans différence notable avec ceux d'A.casaticus.

Uropodes : Un peu plus longs que la moitié du pléotelson; les trois articles ont des longueurs relatives normales : 1-1,25-1,5.

Pléotelson : Comme celui de cavaticus, subcirculaire, presqu'aussi large que long avec pointe du telson faiblement marquée et très arrondie.

La grotte de la Fontaine Saint-Joseph devient la quatrième station connue de valdensis ; on peut penser en effet, d'après le dessin de l'endopodite du pléopode II d'un mâle de la grotte de la Balme (Racovitza in Chappuis 1949, page 85) que les individus de cette caverne appartiennent aussi à cette sous-espèce comme l'a signalé Remy en 1951 ; une étude d'autres exemplaires permettrait de l'établir définitivement. D'ailleurs les deux cavités sont très proches l'une de l'autre et leurs rivières doivent appartenir au même réseau hydrographique. Découvert du Jura Vaudois à la source de l'Orbe, valdensis semble donc avoir une aire de dispersion qui s'étend jusqu'au Jura méridional. Par sa présence à la résurgence du Bief Noir près de Saint-Claude qui constitue ainsi une station intermédiaire, cette sousespèce apparaît comme typiquement jurassienne.

La découverte de la nouvelle forme puteanus et celle d'une nouvelle station de valdensis nous incitent à penser, qu'en France, l'espèce cavaticus est plus différenciée qu'on ne le croyait; à cet effet, 
il serait souhaitable que les Aselles signalés de plusieurs grottes des Alpes dauphinoises (Ginet 1961) et rapportés à l'espèce cavaticus soient réexaminés d'une manière plus approfondie en tenant compte des critères essentiels énumérés ci-dessus. D'autre part, comme le nombre des stations françaises connues est encore assez restreint, il est indispensable que des prospections intensives soient faites dans toutes les régions qui s'étendent des Ardennes au Sud de la France, tant dans le domaine hypogé que dans les sources rhéocrènes et les puits afin, qu'après un examen très précis, l'on puisse, tout au moins en ce qui concerne la faune française, se faire une idée de la variabilité de l'espèce casaticus dans notre pays.

\section{RÉSUMÉ}

En Europe, Asellus casaticus Leydig était jusqu'en 1963 la seule espèce hypogée connue d'une lignée qui s'étend de l'Angleterre jusqu'en Autriche. Jusqu'aux travaux de Racovitza en 1919, tous les Aselles du domaine souterrain étaient rapportés à cette espèce, premier Aselle cavernicole connu. Les critères taxonomiques définis par l'auteur précédent permirent à plusieurs chercheurs de distinguer différentes sous-espèces. Par la suite, devant la variabilité apparente des caractères, Chappuis renonce à nommer les différentes formes rencontrées.

Après avoir pris comme type de référence des individus de la grotte de Sainte-Reine (Meurthe-et-Moselle), qui ont toujours été considérés comme très voisins, sinon identiques au type, nous pensons devoir créer une nouvelle sous-espèce puteanus pour des Aselles d'un puits du Beaujolais. Cette forme diffère de cavaticus f. typ. par la structure de l'organe copulateur du mâle, la forme du pléopode du I mâle etle nombre des épines dactyliennes aux péréiopodes.

Enfin, nous donnons une description plus détaillée de la sous-espèce valdensis Chappuis basée sur des spécimens d'une grotte du Plateau de Crémieu (Isère).

\section{ZUSAMMENFASSUNG}

Bis 1963 war Asellus cavaticus Leydig die einzige bekannte unterirdische Assel einer Stammart, deren europäisches Verbreitungsgebiet sich von England bis Österreich erstreckt. Bis zu den Arbeiten Racovitzas aus dem Jahre 1919 wurden alle Grundwasserasseln jener Spezies zugerechnet, der ersten bekannten unterirdischen Assel. Die von Racovitza festgelegten systematischen Merkmale erlaubten mehreren Forschern, verschiedene Subspezies aufzustellen. Chappuis verzichtet in Anbetracht der bedeutenden Variabilität der systematischen Charaktere auf die Benennung der verschiedenen von ihm aufgefundenen Formen.

Bezugnehmend auf Individuen der Höhle von Sainte-Reine bei Toul - Individuen, die stets als dem Typus sehr nahe stehend oder sogar als mit ihm identisch angesehen wurden - wird die Beschreibung einer neuen Subspezies erforderlich, und zwar für Exemplare aus einem Brunnen des Beaujolais. 
Diese Form unterscheidet sich von cavaticus f. typ. durch die Endorgane der 2. Pleopoden-Endopoditen des Männchens, die Form seiner 1.Pleopoden und die Anzahl der Stacheln am Unterrand der Thorakalbeindactylopoditen.

Ferner wird eine ausführlichere Beschreibung der Unterart valdensis Chappuis für Exemplare aus einer Höhle des Plateaus von Crémieu (Isère) gegeben.

\section{B I B LI O GRAP H I E}

Arcangeli, A. (1935) - Asellus delle caverne del Belgio. Bull. Mus. roy. Hist. Nat. Belgique, 11 : 1-10.

Birstein, J. A. (1951) - Faune de l'URSS, VII/5 Asellota. Moscou : 1-142.

Blanc, H. (1879) - Isopode aveugle de la région profonde du Léman. Asellus foreli sp.nov. Bull. Soc. vaudoise Sc.nat., 16 : 377-394.

Braga, J. M. (1948) - Sur les Asellus de l'Afrique du Nord. Ann. Fac. Ciencias Porto, $33: 1-39$.

Chappuis, P. A. (1948) - Copépodes, Syncarides et Isopodes des eaux phréatiques de Suisse. Rev. Suisse Zool., 55 : 549-566.

- (1949) - Les Asellides d'Europe et pays limitrophes. Arch. Zool. exp. gén. Paris, $86: 78-94$.

- (1953) - Sur la systématique du genre Asellus. Notes biospéol., Paris, $8: 67-79$.

- (1955) - Remarques générales sur le genre Asellus et description de quatre espèces nouvelles. Notes biospéol., Paris, 10 : 163-182.

Dudich, E. (1925) - Systematische Studien an italienischen Aselliden. Ann. Mus. Nat. Hungarici, 22 : 281-301.

Ginet, R. (1952) - La grotte de La Balme (Isère) ; topographie et faune. Bull. Soc. Linn. Lyon, $21: 27-31$.

- $(1953 a)$ - Faune cavernicole du Jura méridional et des chaines subalpines dauphinoises. I. Crustacés aquatiques. Notes biospéol., Paris, 8 : $185-198$.

- $(1953 b)$ - Contribution à la connaissance de la faune cavernicole du Jura méridional. Influence des glaciations quaternaires. C.R. $1^{\text {er }}$ Cong. Intern. Spéléo., Paris, $3: 125-130$.

- (1961) - Faune cavernicole du Jura méridional et des chaines subalpines dauphinoises. II. Contribution à la connaissance des Invertébrés. Ann. Spéléo., Paris, $16: 303-325$.

Henry, J.-P. (1963) - Premier Aselle cavernicole de Basse-Provence : Asellus synaselloides n.sp. Ann. Spéléo., Paris, $18: 99-106$.

Husson, R. (1938) - La faune des galeries de mines de l'Est de la France.

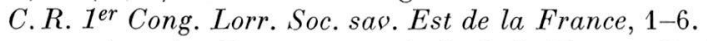

Jeannel, R. (1926) - Faune cavernicole de la France. Paris, P. Lechevalier éd., 1-334.

Karaman, S. (1933) - Über zwei neue Isopoden der Gruppe Asellus aus Jugoslawien. Recueil de travaux offert à Ziv. Gjorjevic, Beograd.

- (1934) - Weitere subterrane Aselliden aus Jugoslawien. Zool. Anz., 108 : $23-26$.

- (1955) - Asellus casaticus Schiödte und seine Nächstverwandten. Acta Mus. macedonici Sc. Nat., 2:1-36. 
LEy IG, F. (1878) - Über Amphipoden und Isopoden. Zeitschr. f. wiss. Zool., $30: 225-274$.

Mifthe, C. (1899) - Asellus cavaticus Schiödte. Ein Beitrag zur Höhlenfauna der Schweiz. Reo. Suisse Zool., 7 : 269-319.

Racovitza, E. G. - (1919) - Notes sur les Isopodes. 1. Asellus aquaticus auct. est une erreur taxonomique. 2. Asellus aquaticus L. et A.meridianus n.sp. Arch. Zool. exp. gén., $58: 31-53$.

- (1920) - Notes sur les Isopodes. 6. Asellus communis Say. 7. Les pléopodes I et II des Asellides, morphologie et développement. Arch. Zool. exp. gén., $58: 79-115$.

- (1922) - Description de trois Asellus (Isopodes) cavernicoles nouveaux. (note préliminaire). Bull. Soc. Sc. Cluj, $1: 401-410$.

Remy, P. (1941) - Asellotes de Yougoslavie et de Grèce. Arch. Zool. exp. gén., Paris, $82: 1-25$.

- (1948) - Sur quelques Crustacés cavernicoles d'Europe. Notes biospéol., Paris, $3: 35-47$.

- (1951) - Stations de Crustacés obscuricoles. Arch. Zool. exp. gén., Paris, $88: 217-230$.

Stammer, H. J. (1932) - Zur Kenntnis der Verbreitung und Systematik der Gattung Asellus, insbesondere der mitteleuropäischen Arten (Isopoda). Zool. Anz., 99 : 113-131.

Strouhal, H. (1957) - Asellus (Prosaellus) im nördlichen Österreich (Isopoda, Asellota). Ann. natur. Mus. Wien, 62 : 263-283.

Tattersall, W. M. (1930) - Asellus casaticus Schiödte, a blind Isopod new to the british Fauna, from a well in Hampshire. Journ. Linn. Soc., $37: 79-91$.

Viré, A. (1904) - La faune souterraine du puits de Padirac (Lot). C.R. Acad. Sc. Fr., 826-828.

\section{EXPLICATIONS DES PLANCHES $9(1)$ et $10(2)$ PLANCHE 9 (1)}

Asellus cavaticus puteanus n.subsp. d'un puits situé au hameau de la Creuze au Pérréon (Rhône).

Fig. A : Extrémité terminale d'une antennule droite d'une.+

Fig. B : Dactyle du péréiopode I droit d'un ô.

Fig. C : Dactyle du péréiopode I gauche d'une o.

Fig. D : Extrémité distale du péréiopode IV gauche d'un ô.

Fig. E : Pléopode I ô droit.

Fig. F : Pléopode II ơ droit.

Fig. G : Extrémité terminale de l'endopodite du pléopode II ơ droit.

Fig. H : Pléopode II 우 droit.

Fig. I : Pléopode III gauche d'un ot-identique chez les 2 sexes.

Fig. J : Pléotelson d'un ô. 


\section{PLANCHE 10 (2)}

Asellus cavaticus valdensis Chappuis, de la grotte de la Fontaine SaintJoseph à Verna (Isère).

Fig. A : Epipodite et oostégite du maxillipède gauche d'une o o ovigère.

Fig. B : Dactyle du péréiopode I droit d'un đo.

Fig. C : Dactyle du péréiopode I gauche d'une o.

Fig. D : Extrémité distale du péréiopode IV gauche d'un ơ.

Fig. E : Pléopode I ơ droit.

Fig. F : Pléopode II $\sigma^{\star}$ droit:

Fig. G : Extrémité distale de l'endopodite du pléopode II ô droit.

Fig. H : Pléopode II 9 droit.

Fig. I : Pléopode III gauche d'un $\widehat{o}$-identique chez les 2 sexes. 
SPELEOLOGY II

(Henry, 1)

PLATE 9


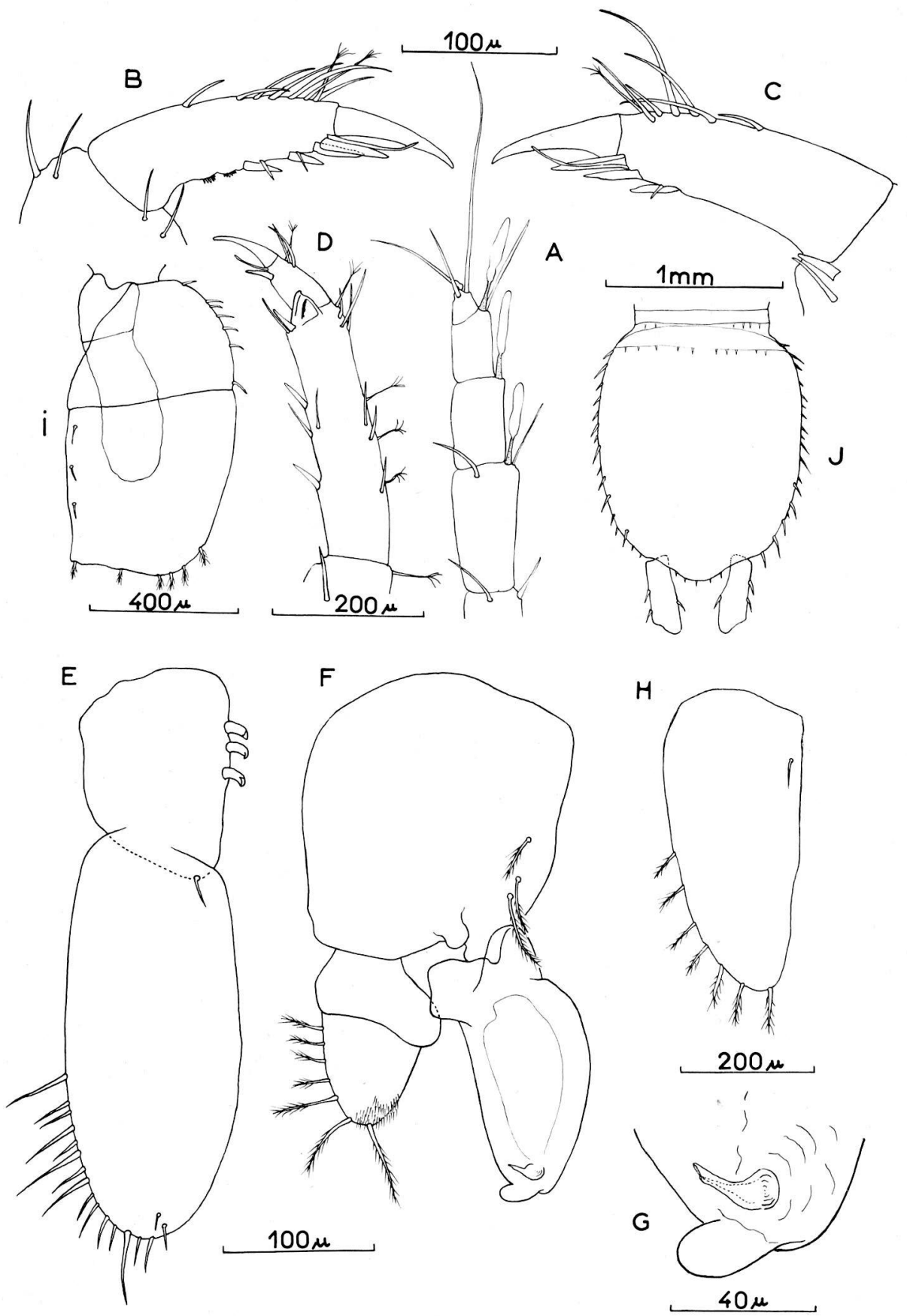